\title{
Monitoring feeding behaviour of dairy cows using accelerometers
}

\author{
Gabriele Mattachini, Elisabetta Riva, Francesca Perazzolo, Ezio Naldi, Giorgio Provolo \\ Department of Agricultural and Environmental Sciences, University of Milan, Italy
}

\begin{abstract}
Monitoring cow behaviour has become increasingly important in understanding the nutrition, production, management of the well being, and overall health of dairy cows. Methods of assessing behavioural activity have changed in recent years, favouring automatic recording techniques. Traditional methods to measure behaviour, such as direct observation or time-lapse video, are labour-intensive and time-consuming. Automated recording devices have become increasingly common to measure behaviour accurately. Thus, the development of automated monitoring systems that can continuously and accurately quantify feeding behaviour are required for efficient monitoring and control of modern and automated dairy farms. The aim of this study was to evaluate the possible use of a 3D accelerometer to record feeding behaviour of dairy cows. Feeding behaviour (feeding time and number of visits to the manger) of 12 lactating dairy cows was recorded for approximately $3 \mathrm{~h}$ with $3 \mathrm{D}$-accelerometer data loggers (HOBO Pendant G logger). The sensors were positioned in the high part of the neck to monitor head movements. Behaviour was simultaneously recorded using visual observation as a reference. Linear regression analysis between the measurement methods showed that the recorded feeding time $\left(\mathrm{R}^{2}=0.90, \mathrm{n}=12, \mathrm{P}<0.001\right)$ was closely related to visual observations. In contrast, the number of visits was inadequately recorded by the 3D-accelerometer, showing a poor relationship with visual observations $\left(\mathrm{R}^{2}=0.31, \mathrm{n}=12, \mathrm{P}<0.06\right)$. Results suggest that the use of accelerometer sensors can be a reliable and suitable technology for monitoring feeding behaviour of individual dairy cows in free stall housing. However, further research is neces-
\end{abstract}

Correspondence: Gabriele Mattachini, Department of Agricultural and Environmental Sciences, University of Milan, via G. Celoria 2, 20133 Milan, Italy.

Tel.: +39.02.5031.6858 - Fax: +39.02 .5031 .6845 .

E-mail: gabriele.mattachini@unimi.it

Key words: Accelerometer sensor; automatic monitoring system; dairy management; cow feeding behaviour.

Contributions: the authors contributed equally.

Received for publication: 14 July 2015.

Accepted for publication: 13 November 2015.

(C) Copyright G. Mattachini et al., 2016

Licensee PAGEPress, Italy

Journal of Agricultural Engineering 2016; XLVII:498

doi:10.4081/jae.2016.498

This article is distributed under the terms of the Creative Commons Attribution Noncommercial License (by-nc 4.0) which permits any noncommercial use, distribution, and reproduction in any medium, provided the original author(s) and source are credited. sary to develop an appropriate device able to detect and recognise the movements connected with the head movement during feeding. Such a device could be part of an automatic livestock management tool for the efficient monitoring and control of comfort and welfare of dairy cows under the intensive conditions of modern automated dairy farms.

\section{Introduction}

Monitoring cow behaviour has become increasingly important in understanding the nutrition, production, management of the well being, and overall health of dairy cows. In the last few years, an interest in the feeding behaviour has resulted in a better understanding of the effect of management and the physiological state of dairy cows. Daily activity patterns, eating, and ruminating are widely considered as indicators closely related to health issues (Huzzey et al., 2007; Weary et al., 2009) and productivity for individual dairy cows.

Feeding behaviour of dairy cows traditionally has been determined using intensive research procedures, such as direct observation and time-lapse video recording (Overton et al., 2002). These methods are very labour-intensive and time-consuming (Müller and Schrader, 2003; Elischer et al., 2013), which limits their use over long time periods and for many animals. Methods of assessing behavioural activity have changed in recent years, favouring automatic recording techniques. Automated recording devices for measuring feeding behaviour and feed intake of cattle are being used more and more widely (Krawczel et al., 2012; Bikker et al., 2014; Chizzotti et al., 2015). The development of automated monitoring systems that can continually and accurately quantify feeding behaviour could provide an early warning tool for efficient monitoring and control of modern and automated dairy farms.

The objective of this study was to evaluate the possible use of a 3Daccelerometer, installed to the neck collar, to record feeding behaviour of dairy cows, compared to direct visual observation.

\section{Materials and methods}

\section{Housing, animals and management}

The study was conducted between June and July 2013 on the experimental farm A. Menozzi (Landriano, Italy; 45 19' 16.5” N, 9 15’ 56.4” E) of the University of Milan. A total of 12 lactating Holstein Friesian dairy cows were used, divided into eight multiparous [parity $=3.5 \pm 1.1$, days in milk $=220.1 \pm 102.7$, milk yield $=27.6 \pm 7.5 \mathrm{~kg} / \mathrm{d}$; mean \pm standard deviation (SD)] and four primiparous cows (days in milk $=225.8 \pm 40.1$, milk yield $=27.8 \pm 3.9 \mathrm{~kg} / \mathrm{d}$; mean $\pm \mathrm{SD}$ ). Cows were housed in a free stall pen in a loose-housing layout with a total of 135 cubicles having rubber mats and 132 feeding places. A total mixed ration was delivered once daily beginning at approximately 10:00 h. Water was provided ad libitum. Cows were milked two times daily at approximately 09:00 a.m. and 09:00 p.m. Stalls and alleyways were cleaned once daily at approximately 09:30 a.m.. 


\section{Data collection}

Feeding behaviour of the 12 lactating dairy cows was monitored simultaneously with 3D-accelerometers (HOBO Pendant G data logger; Onset Computer Corp., Pocasset, MA, USA) and visual observation. Behavioural data (feeding time and number of visits to the manger) of each cow was collected continuously for $3 \mathrm{~h}$, in two 1.5-h blocks each. The two 1.5-h blocks were recorded at different times (approximately from 10:00 to 11:30 h, and from 13:00 to 14:30 h). This procedure prevented time-specific behaviours from dominating the dataset and provided raw data that represented different levels of activity.

Two observers having extensive previous animal monitoring were trained to classify the visual observations of feeding behaviour. Direct visual observation was arranged in a time-interval of 1-s scan samplings. The following feeding behaviour variables were measured: i) number of visits (n), defined as the total number of times a cow's nose is located in or above the feed and makes a chewing or gathering movement from the feed bunk; ii) feeding time (min), defined as the summation of the duration of all visits to the feed bunk.

The 3D-accelerometer is a waterproof, 3-channel data logger with 8bit resolution, and can record up to approximately 21,800 combined $\mathrm{x}$-, $\mathrm{y}-$, and z-axis acceleration readings or internal logger events. The logger uses a coupler and optical base station with USB interface to transfer data to a computer. The 3D-accelerometers were attached to the topside of the neck collars of the cows using an aluminium support, in a position such that the $\mathrm{x}$-axis and $\mathrm{y}$-axis were parallel to the cow's neck and perpendicular to the ground, respectively, and the z-axis was perpendicular to the cow's neck. The 3D-accelerometers were programmed to record the acceleration and tilt angle (calculated by static accelerations due to gravity) on the $\mathrm{x}-, \mathrm{y}-$, and $\mathrm{z}$-axes at $2-\mathrm{Hz}$ intervals. The $3 \mathrm{D}$ accelerometer data were downloaded using Onset HOBOware software version 3.7.1 (Onset Computer Corp.). These data were exported into a Microsoft Excel ${ }^{\mathrm{TM}} 2007$ spread sheet. The degree of horizontal tilt (xaxis) was used to measure the head movements of the cow and determine the feeding behaviour as feeding time and number of visits. The threshold values used to categorise tilt angle readings as a specific head tilt were determined based on normal angle of head movements. The $\mathrm{x}$-axis tilt readings $>110^{\circ}$ indicated the cow's head was tilted (gathering of feed), whereas readings $\leq 110^{\circ}$ indicated the cow's head was horizontal (chewing movements after gathering). A gathering-offeed event was identified when the cow's head was tilted continuously for at least $10 \mathrm{~s}$ but not more of $60 \mathrm{~s}$; otherwise, the maximum interval between two gathering events or maximum duration of chewing movements after gathering was $60 \mathrm{~s}$. The thresholds used to categorise $\mathrm{x}$ axis tilt readings as specific behaviours (gathering and chewing) were determined based on preliminary visual observation from the video recordings. For example, if the cow's head was tilted for more than 60 $\mathrm{s}$ or less than $10 \mathrm{~s}$, these readings were observed to be likely associated with head movements outside of feeding behaviour (head tilted near cubicles or social behaviour). The sequence and the duration of these two head positions (tilted and horizontal) were used to evaluate the feeding time, which was calculated as the summation of the duration of the head tilted (gathering of feed) and head horizontal (chewing movements after gathering). A visit event was considered only with two consecutive gathering-of-feed events.

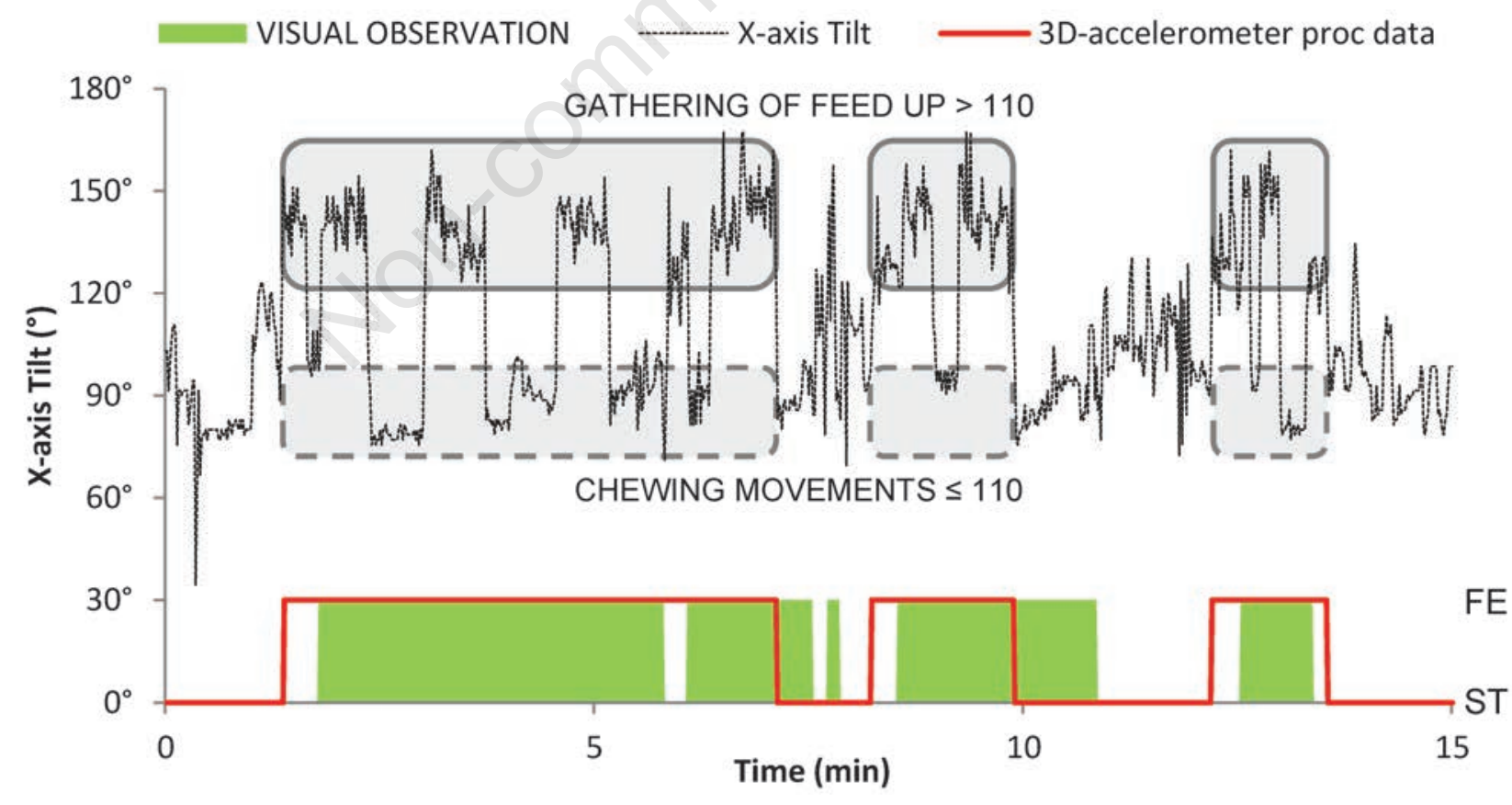

Figure 1. Feeding behaviour pattern of a single cow (Cow 5) during a 15-min observation period recorded by visual observation, 3Daccelerometer raw data (x-axis tilt), and 3D-accelerometer processed data with a sampling interval of 1 s. Behaviours recorded by visual observation were as follows: feeding behaviour (FE, comprised of feeding time and number of visits) and standing (ST, comprised of other behaviours except feeding). Dashed boxes indicate the gathering of feed ( $x$-axis tilt $\left.>110^{\circ}\right)$ and the chewing movements $(x$-axis tilt $\leq 110^{\circ}$ ) recorded by $3 \mathrm{D}$-accelerometer. 


\section{Statistical data analysis}

Feeding behaviour (number of visits and feeding time) data were analysed on an hourly basis to facilitate comparisons between the 3Daccelerometer and direct visual observations. Estimates of feeding time (min) and the number of visits (n) for each cow obtained from the 3Daccelerometer (as the dependent variable) were compared with the values obtained from the direct visual observations (as the independent variable) using the linear regression procedure in SAS (PROC REG; SAS Institute Inc., Cary, NC, USA). To determine the agreement between the $3 \mathrm{D}$-accelerometers and direct visual observation feeding behaviour data (at a $2-\mathrm{Hz}$ interval) were analysed using $2 \times 2$ contingency tables (FREQ procedure of SAS; SAS Institute Inc.). Observed cow feeding behaviour (i.e., cow truly at the manger) - that was correctly recorded as feeding behaviour by the 3D-accelerometer - was assigned a true positive (TP). Observed cow feeding time that was not recorded correctly by the $3 \mathrm{D}$-accelerometer was assigned a false negative (FN). Observed cow non-feeding behaviour (i.e., cow not at the manger) that was correctly recorded as non-feeding behaviour by the 3D-accelerometer was assigned a true negative (TN), and observed non-feeding behaviour that was recorded as feeding behaviour by the 3D-accelerometer was assigned a false positive (FP). The sensitivity $[\mathrm{Se}=\mathrm{TP} /(\mathrm{TP}+\mathrm{FN})$; i.e. , the proportion of true positives that were correctly identified by the $3 \mathrm{D}$-accelerometer] and specificity [Sp $=\mathrm{TN} /$ (TN + FP); i.e., proportion of true negatives that were correctly identified by the $3 \mathrm{D}$-accelerometer] were determined by treating the direct visual observations as the gold standard (Altman and Bland, 1994a). Predictive values were calculated as the probability of correct positive identifications by the 3D-accelerometer $[\mathrm{PPV}=\mathrm{TP} /(\mathrm{TP}+\mathrm{FP})]$ and probability of negative identifications $[\mathrm{NPV}=\mathrm{TN} /(\mathrm{TN}+\mathrm{FN})]$ (Altman and Bland, 1994b).

\section{Results and Discussion}

Examples of the feeding behaviour pattern of a single cow (Cow number 5), obtained from visual observation, as well as from raw data recorded and processed by the 3D-accelerometer, are shown in Figure 1. In this figure, the feeding time and number of visits enumerated by visual observation are highlighted as green areas, and are compared with those identified by 3D-accelerometer processed data. In addition, the gathering-of-feed and chewing movements detected by the 3Daccelerometers are marked with dashed boxes. For Cow number 5 , the 3D-accelerometer determined three feeding visits and 8.7 min of feeding time, as well as 11 gathering-of-feed movements ( $\mathrm{x}$-axis tilt $>110^{\circ}$ ) and eight chewing movements ( $\mathrm{x}$-axis tilt $\leq 110^{\circ}$ ), compared to five visits and $8.8 \mathrm{~min}$ of feeding time identified by the visual observation.

By direct visual observation, the number of visits by an individual cow ranged from 3 to 29 (mean of 12.3), and feeding time ranged from 4.40 to $111.30 \mathrm{~min}$ (mean of $47.53 \mathrm{~min}$ ) (Table 1). Sensitivity, specificity and predictive values (positive and negative) for the feeding time observations between 3D-accelerometer processed data and direct visual observation also are reported in Table 1. The 3D-accelerometer provided data of high sensitivity $(\mathrm{Se}=0.789)$ and specificity $(\mathrm{Sp}=0.937)$ for feeding time, and also displayed high predictive values ( $\mathrm{PPV}=0.819$ and NPV=0.925). These results indicated that the 3D-accelerometers had a near- $80 \%$ probability of correctly identifying feeding time. However, there was a large standard deviation of mean sensitivity, which indicated greater among-cow variability for the feeding time detection. In fact, despite the high sensitivity, specificity and probability, data for some monitored cows highlighted a large error or difference between the values of feeding time and number of visits obtained by $3 \mathrm{D}$-accelerometer compared to the values obtained by visual obser-

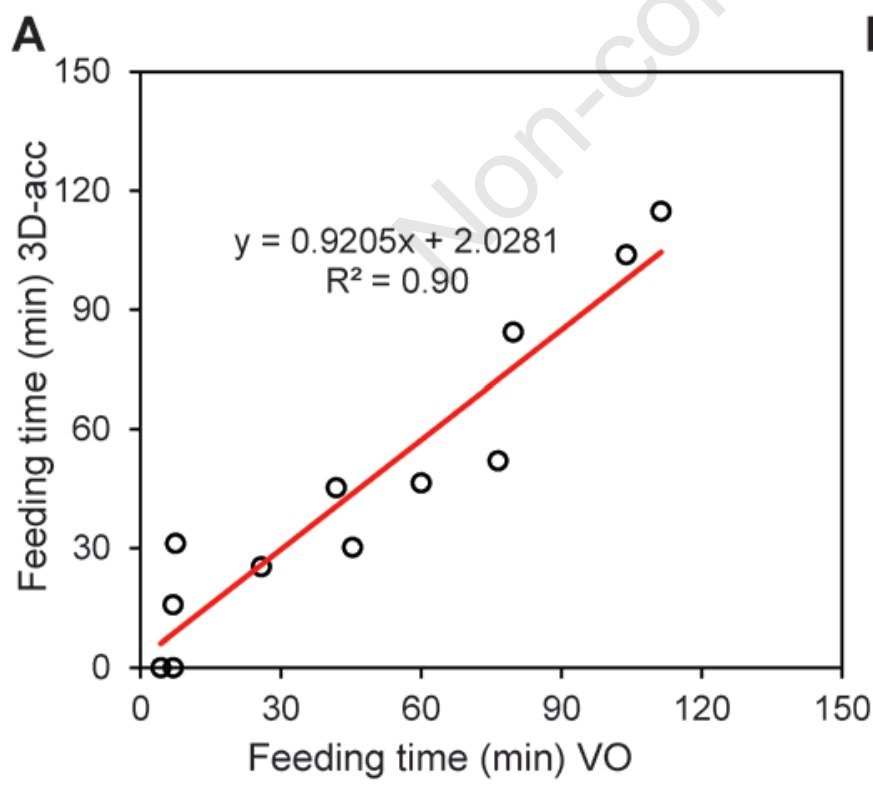

B

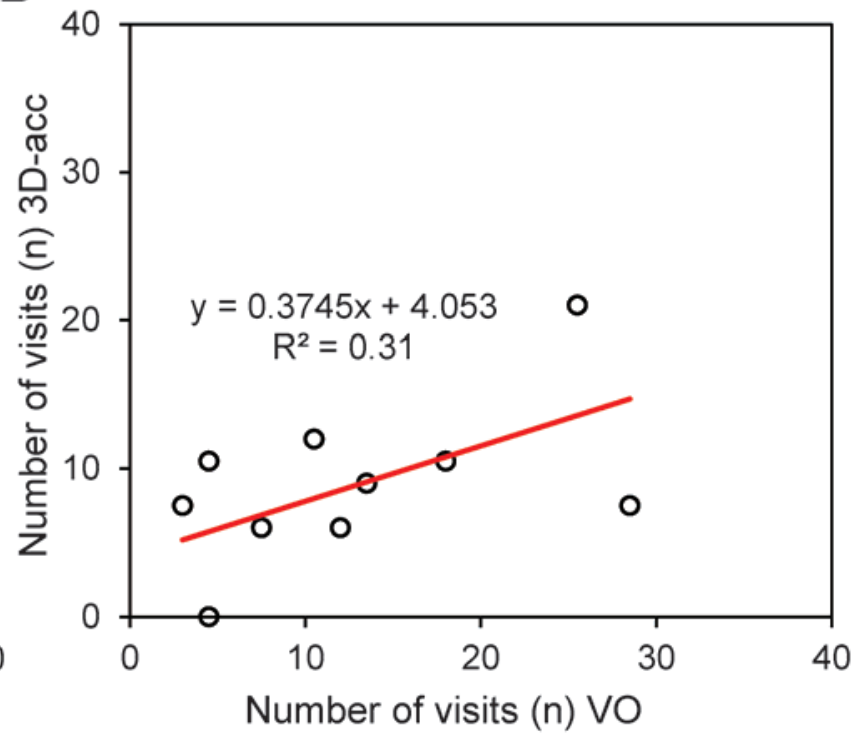

Figure 2. Relationships between the 3D-accelerometer (3D-acc) (dependent variable) and the direct visual observations (VO) (independent variable), based on 12 cows monitored over $3 \mathrm{~h}$. (A) Correlation of feeding time (min), and (B) correlation of the number of visits (n). 
Table 1. Mean values for number of visits and feeding time of 12 cows monitored for 3 hours by visual observation and 3D-accelerometer processed data. Sensitivity, specificity, positive and negative predictive values from $2 \times 2$ contingency tables for the feeding time observations between 3D-accelerometer processed data and direct visual observation.

\begin{tabular}{|c|c|c|c|c|c|c|c|c|}
\hline \multirow[b]{2}{*}{ No. Cow } & \multicolumn{2}{|c|}{ Number of visits (n) } & \multicolumn{2}{|c|}{ Feeding time (min) } & \multicolumn{4}{|c|}{$\begin{array}{c}\text { 3D-accelerometer-VO } \\
\text { Feeding time }\end{array}$} \\
\hline & VO & 3D-acc & VO & 3D-acc & Se & Sp & PPV & NPV \\
\hline 1 & 29 & 8 & 76.45 & 52.05 & 0.566 & 0.915 & 0.831 & 0.741 \\
\hline 2 & 14 & 9 & 45.35 & 30.24 & 0.442 & 0.924 & 0.663 & 0.831 \\
\hline 3 & 12 & 6 & 25.86 & 25.32 & 0.829 & 0.975 & 0.846 & 0.971 \\
\hline 4 & 26 & 21 & 41.88 & 45.30 & 0.783 & 0.909 & 0.724 & 0.932 \\
\hline 5 & 11 & 12 & 79.70 & 84.31 & 0.894 & 0.870 & 0.845 & 0.912 \\
\hline 6 & 8 & 6 & 103.90 & 103.86 & 0.968 & 0.957 & 0.968 & 0.956 \\
\hline 7 & 3 & 8 & 6.95 & 15.78 & 0.966 & 0.948 & 0.426 & 0.999 \\
\hline 8 & 5 & 11 & 7.53 & 31.23 & 0.983 & 0.862 & 0.237 & 0.999 \\
\hline 9 & 11 & 12 & 60.01 & 46.50 & 0.656 & 0.941 & 0.847 & 0.846 \\
\hline 10 & 18 & 11 & 111.30 & 114.78 & 0.962 & 0.887 & 0.932 & 0.934 \\
\hline 11 & 5 & 0 & 4.40 & 0.00 & 0.000 & 1.000 & 0.000 & 0.976 \\
\hline 12 & 5 & 0 & 7.02 & 0.00 & 0.000 & 1.000 & 0.000 & 0.961 \\
\hline All & 12.3 & 8.7 & 47.53 & 45.78 & 0.789 & 0.937 & 0.819 & 0.925 \\
\hline
\end{tabular}

VO, visual observation; 3D-acc, 3D-accelerometer; Se, sensitivity; Sp, specificity; PPV, positive predictive value; NPV, negative predictive value.

vation (Table 1).

The linear regression analysis confirmed the clear relationship between the 3D-accelerometer and direct visual observation for feeding time $\left(\mathrm{R}^{2}=0.90, \mathrm{n}=12, \mathrm{P}<0.001\right.$; Figure 2$)$. In contrast, the number of visits was inadequately detected by the $3 \mathrm{D}$-accelerometers, as indicated by the very poor relationship with visual observations $\left(R^{2}=0.31, n=12\right.$, $\mathrm{P}<0.06$; Figure 2).

The use of automatic recording devices to measure feeding behaviour has become increasingly common, as the devices record non-invasively and overcome the time-consuming limitations of visual-based observations. Automatic monitoring of dairy cow feeding behaviour could improve dairy herd management. The current study demonstrated that the 3D-accelerometer could measure reliably the feeding time (compared to direct visual observation) in lactating dairy cows in a loose-housing system. The linear regression analysis showed close relationships between the feeding time identified by the 3D-accelerometer and by visual observation. The capability of the $3 \mathrm{D}$-accelerometer to measure the feeding time was confirmed by $2 \times 2$ contingency tables. The inconsistency highlighted in data for some monitored cows most likely was due to a notable inter-individual variability between cows, different sensitivity to the threshold values selected to identify natural head tilt position, and from a small displacement of the data logger aluminium support during the monitoring (which modified the position of $3 \mathrm{D}$-accelerometer respect to the cow's neck axis). The results from this study are similar to those from validation studies using video or visual observations as a control, which have shown high levels of correspondence between video recording and automatic monitoring devices when considering the total duration of behavioural activities (Müller and Schrader, 2003; Büchel and Sundrum, 2014; Chizzotti et al., 2015).

In contrast to the results for feeding duration, the probability that the 3D-accelerometer correctly recorded the number of feeding visits was low. Thus, the linear regression analysis showed a lower correlation between the 3D-accelerometer data and visual observations for visits than for feeding duration. In general practice, the number of visits made by a cow to the feed bunk is limited, and visits are difficult to classify; thus, a more precise measuring method is required than for monitoring feeding time. The thresholds used to categorise $\mathrm{x}$-axis tilt readings probably affected the accuracy of counting visits to the feed bunk more than measuring feeding time because feeding visits depend largely on the individual behaviour of the cows, and not only on the range of the head tilt.

Improvements in data processing might enhance the performances of this device. A more comprehensive algorithm supporting real time analysis of recorded data and improving the filtering procedure could be a more accurate way to estimate the number of visits, and at the same time improve detection of the feeding time. Moreover, further research using more cows and more observational days (to reduce the effect of inter-individual variability between cows) is needed to improve and verify the model and the algorithm that was used to process 3D-accelerometer data.

\section{Conclusions}

Results from this study support the conclusion that the use of accelerometer sensors can be a reliable and suitable technology for monitoring feeding behaviour of individual dairy cows in free stall housing. However, further research is necessary to develop an appropriate device able to detect and recognise the movements connected with the head movement during feeding. Such a device could be part of an automatic livestock management tool for the efficient monitoring and control of comfort and welfare of dairy cows under the intensive conditions of modern automated dairy farms.

\section{References}

Altman D.G., Bland J.M. 1994a. Diagnostic tests 1: sensitivity and specificity. Brit. Med. J. 308:1552.

Altman D.G., Bland J.M. 1994b. Diagnostic tests 2: predictive values. Brit. Med. J. 309:102.

Bikker J.P., van Laar H., Rump P., Doorenbos J., van Meurs K., Griffioen G.M., Dijkstra J. 2014. Technical note: evaluation of an earattached movement sensor to record cow feeding behavior and activity. J. Dairy Sci. 97:2974-9.

Büchel S., Sundrum A. 2014. Technical note: evaluation of a new sys- 
tem for measuring feeding behavior of dairy cows. Comput. Electron. Agric. 108:12-6.

Chizzotti M.L., Machado F.S., Valente E.E., Pereira L.G., Campos M.M., Tomich T.R., Coelho S.G., Ribas M.N. 2015. Technical note: validation of a system for monitoring individual feeding behavior and individual feed intake in dairy cattle. J. Dairy Sci. 98:3438-42.

Elischer M.F., Arceo M.E., Karcher E.L., Siegford J.M. 2013. Validating the accuracy of activity and rumination monitor data from dairy cows housed in a pasture-based automatic milking system. J. Dairy Sci. 96:6412-22.

Huzzey J.M., Veira D.M., Weary D.M., von Keyserlingk M.A.G. 2007. Prepartum behavior and dry matter intake identify dairy cows at risk for metritis. J. Dairy Sci. 90:3220-33.
Krawczel P.D., Klaiber L.M., Thibeau S.S., Dann H.M. 2012. Technical note: data loggers are a valid method for assessing the feeding behavior of dairy cows using the Calan Broadbent Feeding System. J. Dairy Sci. 95:4452-6.

Müller R., Schrader L. 2003. A new method to measure behavioural activity levels in dairy cows. Appl. Anim. Behav. Sci. 83:247-58.

Overton M.W., Sischo W.M., Temple G.D., Moore D.A. 2002. Using timelapse video photography to assess dairy cattle lying behavior in a free-stall barn. J. Dairy Sci. 85:2407-2413.

Weary D.M., Huzzey J.M., von Keyserlingk M.A.G. 2009. Board-invited review: Using behavior to predict and identify ill health in animals. J. Anim. Sci. 87:770-7. 